\title{
Demographically Corrected Norms
}

\section{A Case Illustration of an Unmet Need in High-Stakes Testing Among Native Americans}

\author{
Jennifer L. Callahan \\ Oklahoma State University, Stillwater
}

\begin{abstract}
Since the United States Supreme Court ruling in 2002 on the Atkins v. Virginia case, which determined that executing an individual with mental retardation violates the Eighth Amendment, the potential motivation to malinger mental retardation by individuals charged in potential death penalty cases has emerged. The following case illustrates some of the challenges that emerge in such an evaluation, with particular attention on the impact of inequitable standardization for demographically adjusted norms.
\end{abstract}

Keywords: Native American; American Indian; Alaskan Native; demographic norms; malingering; mental retardation

\section{Theoretical and Research Basis}

In 2002, the U.S. Supreme Court determined that execution of a mentally retarded individual is a violation of the Eighth Amendment (Atkins v. Virginia, 2002), which prohibits excessive punishment. Since that time, many state legislatures have enacted statutes offering sentencing protection for any individual with mental retardation. Of course, evidence of mental retardation must be presented for the court's consideration to support such a claim and such evidence often includes the results of psychological testing.

Competent psychological evaluation rightly demands conscientious consideration of the obtained data with an appreciation of possible moderating variables. For that reason, demographically corrected norms were consulted during the conceptualization of the following case. However, it was found that relevant demographic corrections on the Wechsler Adult Intelligence Scale-III (WAIS-III; Wechsler, 1997a) and Wechsler Memory Scale-III (WMS-III; Wechsler, 1997b) were not available because of the client's Native American ethnicity. This case presentation is intended to stimulate discussion on the issue of demographic corrections, particularly since revisions of the WAIS-III and WMS-III tests are anticipated within the next few years.

The necessary conditions for documenting mental retardation in forensic cases varies somewhat by state, although most states draw from the definitions provided by professional organizations, including the American Association on Mental Retardation's (AAMR) 1983, 1992, and 2002 formulations and the Diagnostic and Statistical Manual of Mental Disorders (DSM-IV-TR; American Psychiatric Association, 2000) definition. Current conceptualizations by these organizations are consistent in describing mental retardation as being characterized by persistenting significant deficits in both intellectual and adaptive 
functioning (e.g., social skills, conceptual skills, practical skills, etc.), with the onset of problems beginning prior to adulthood.

The negative social connotations and the significantly restricted range of options that can follow diagnosis of mental retardation have historically encouraged speculation that feigning of such a condition would be counterproductive and unlikely. Yet in a relatively recent study by Johnstone and Cooke (2003), it was demonstrated that nonimpaired individuals can willfully produce scores within the mentally retarded range on measures of intelligence. Since the Supreme Court's ruling in the Atkins v. Virgina (2002) case, a clear motivation exists for individuals to simulate mental retardation in death penalty cases. This motivation, coupled with the finding that it is possible to fake mental retardation, brings to the forefront the issue of malingering mental retardation.

Unfortunately, there are no standardized measures available at this time to detect malingering of mental retardation. Given this constraint, evaluators might choose to give other measures for detecting cognitive malingering (e.g., Rey 15-Item Memory Test; Rey, 1964; Test of Memory Malingering [TOMM]; Tombaugh, 1996). However, such measures are meant to detect memory malingering and not cognition more broadly, which limits their use for detecting mental retardation malingering. Johnstone and Cooke's (2003) findings suggest that detection of nonimpaired invidivuals simulating mental retardation may be facilitated by evaluators being alert for behavior functioning that is inconsistent with the obtained test results, subtest inconsistencies, and exaggerated simulation, which may appear as floor effects during testing.

\section{Case Introduction}

Because of the forensic nature of the following case, the evaluation of possible mental retardation included a standard measure of memory malingering as well as a comprehensive battery of neuropsychological tests and procedures. In addition, both the WAIS-III and WMS-III were administered. Predicted scores on the WMS-III were computed from the obtained WAIS-III scores to examine consistency in performance across tests.

\section{Presenting Complaints and Reason for Referral}

Mr. Dexter is a 24-year-old, never married, right-handed Native American male who was referred for neuropsychological evaluation with particular attention to possible mental retardation.

\section{History}

\section{Social and Family History}

Mr. Dexter is the fifth of 8 children born to his mother and has no memory of his father, who died when Mr. Dexter was a young child. Mr. Dexter was reportedly sexually and 
physically abused for several years during middle childhood, ultimately causing him to run away from home. After apprehension, he was placed with a relative for custodial care (at approximately age 11), although his physical placement varied throughout adolescence. Mr. Dexter has never been married and has no children. He remains in contact with the relative that assumed guardianship of him describing that as a good relationship.

\section{Medical History}

No information is available regarding the prenatal care, birth, or early development of Mr. Dexter. His medical history is remarkable because of a motor vehicle accident in his early years, which resulted in ejection from the vehicle in his early teens. However, he denied any history of concussion or other head injury and was able to recall emergency medical service arriving at the scene of the accident.

Mr. Dexter also reported surviving a house fire in his early 20s, which he set. Medical records indicate that fire department personnel discovered Mr. Dexter on the ground outside of the home with $2 \%$ to $3 \%$ of his body burned. During examination in the emergency room, soot within the nose and mouth was noted as was singeing of nasal hair. There was also significant soot within the oropharynx and the oropharynx was erythematous. Admission diagnoses included inhalation burns and burns to his hands and 3\% of total body surface area.

Mr. Dexter was treated in the intensive care unit and became septic. He responded to antibiotic treatment for the sepsis but required a tracheostomy because of continuing pulmonary insufficiency. After a few weeks, he was weaned off artificial respiration and discharged with the diagnoses updated to $2 \%$ body surface area burn injuries involving both upper extremities; pulmonary insufficiency with adult respiratory distress syndrome; and posttraumatic stress disorder.

\section{Educational and Employment History}

According to Mr. Dexter, he did not attend school until age 9. His precise age when formal education was initiated is inconsistently reported in the available records (none younger than age 11), but all records agree that Mr. Dexter was kept from school by his mother for several years. Mr. Dexter stated that he discontinued his education in the 7th grade although incomplete school records are available until the 12th grade. Recurrent psychiatric hospitalizations, suspensions, and changes in school placement appear to have resulted in inconsistent enrollment for much of his secondary education years with very few completed courses or credits toward graduation. Mr. Dexter reports a poor employment history, with only intermittent, unskilled positions and recurring conflicts with both coworkers and supervisors.

\section{Psychiatric History}

Mr. Dexter reported recurrent depressive episodes beginning in childhood with a suicide attempt in middle childhood. He stated that he began experiencing auditory hallucinations during adolescence, including occasional command hallucinations encouraging or demanding him to behave aggressively. He described feeling paranoid and stated a belief that at times he can hear people's thoughts as he walks by them. Such thoughts are uniformly characterized by 
Mr. Dexter as very negative, hostile, and threatening that prompts him to feel anger, frustration, and aggressive urges. Finally, Mr. Dexter noted recurrent intrusive recollections of childhood abuse with pervasive hypervigilance. While undergoing treatment following the house fire, Mr. Dexter was started on an antidepressant and has inconsistently been prescribed medications from the selective serotonin reuptake inhibitors (SSRI) class since that time. At the time of the evaluation, he reported being prescribed an antipsychotic medication, but described inconsistent medication compliance.

\section{Assessment}

\section{Results of Previous Testing}

Mr. Dexter was evaluated shortly after beginning formal education and found to have broad cognitive abilities that were commensurate with the bottom 3 of 1,000 same-aged children (standard score [SS]: 59). Cognitive aptitude for reading (SS: 68), spelling (SS: 69), and mathematics (SS: 62) were all impaired with achievement in each of these areas significantly impaired (broad reading achievement SS: 48; broad written language SS: 28 ; and broad mathematics SS: 55). In contrast, visual integration skills were considered adequate. It was concluded that Mr. Dexter was functioning within the mentally retarded range of abilities.

Re-evaluation of Mr. Dexter approximately 18 months later revealed general intellectual functioning at the lower limit of the borderline impaired range (SS: 71), although it was thought at that time that he might have the potential for low average functioning, with his poor scores hypothesized as reflecting the lack of early academic and intellectual stimulation. At that time, his achievement in word recognition was equivalent to the fourth-grade level (SS: 80), whereas both spelling (SS: 74) and written arithmetic (SS: 65) were approximately equivalent to the third-grade level.

Educational achievement testing was repeated 18 months later with results indicating impaired composite educational achievement (SS: 58). Mr. Dexter's achievement levels in both spelling and arithmetic had not progressed because the previous testing and remained approximately equivalent to third-grade achievement. Rather than testing word recognition, testing of reading more broadly revealed achievement consistent with the second-grade level. His general intellectual functioning was thought to be in the low average range at that time, although substantial subtest variability was observed and learning disability was considered.

\section{Results of Present Testing}

Mr. Dexter's grooming was appropriate to the prison environment and appearance was on par with his chronological age. He was alert throughout the evaluation, although he commented once about fatigue early in testing (he had just woken up). Fund of personal and general knowledge was adequate. Affect was rather flat throughout the evaluation but Mr. Dexter was verbally pleasant and cooperative. He ambulated without assistance or difficulty. Rate of speech, rhythm, and prosody were unremarkable. Mr. Dexter denied experiencing any anxiety pertaining to the evaluation and there was no behavioral indication of noncompliance or symptom magnification. There was no indication of delusional thinking 
at the time of the evaluation. There was no indication of overt hallucinations during the evaluation, although some sensory confusion may have been present. For example, he commented that sometimes he hears people speaking in a foreign language without any apparent recognition that someone really was speaking Spanish within earshot of the evaluative setting. Rapport appeared to be adequately formed, and Mr. Dexter appeared to put forth good effort throughout the evaluation.

\section{Orientation}

Mr. Dexter was alert and oriented for person, place, day of week, and time of day. Although he was able to identify the date of his next court hearing later in the month, he misidentified the month (guessing October rather than June), date (guessing the 20th rather than the 9th), and year (guessing 1999 rather than 2004). He could not identify the reason for referral. The examiner oriented him prior to initiating further cognitive testing. Testing was accomplished during two sessions within the same day. At the conclusion of the evaluation, Mr. Dexter was able to correctly recall the correct date, month, year, and reason for referral.

\section{Test of Motivation}

On the TOMM, Mr. Dexter performed within normal limits on each of two trials, indicating good effort during this portion of cognitive testing.

\section{Attention and Working Memory}

Mr. Dexter's basic auditory attention fell within the mildly impaired range $(T=37)$, with no significant difference between his forward recall (5 digits) and reversed recall (3 digits). Performance on a more complex, auditory working memory task fell in the moderately impaired range $(T=26)$. His overall Working Memory Index score from the WAIS-III $(67 ; T=28)$ fell within the impaired range.

\section{Processing Speed}

Performances were somewhat variable on measures of processing speed. Mr. Dexter obtained an average score on a task of rapid word naming (Stroop Word Trial: $T=52$ ) but a low average score on a similar task of rapid color naming (Stroop Color Trial: $T=42$ ). He again performed in the average range on a task of simple visual tracking and psychomotor speed (Trials A: $T=51)$. However, he obtained a mildly impaired score on a coding task from the WAIS-III (Digit Symbol: $T=37$ ). On a timed task of matching symbols (WAIS-III Symbol Search), Mr. Dexter performed in the moderately impaired range $(T=26)$. The resultant WAIS-III Processing Speed Index score was within the mildly impaired range (PSI $=70, T=30$ ).

\section{Sensory-Perceptual}

No signs of hemispatial neglect, inattention, or extinction were evident across tactile, auditory, or visual systems on incidental behavior. Mr. Dexter displayed a slight tendency 
toward left-sided placement on a task of line bisection but made no omissions and was within normal limits. He made two omission errors on a more visually challenging symbol cancellation task, which borders normal limits.

\section{Motor Functions}

Mr. Dexter reported right-hand dominance. Performances on tests of manual strength were average bilaterally $(T \mathrm{~s}=45)$. Motor speed, as measured by a finger oscillation task, was also average bilaterally $(T \mathrm{~s}=51)$. Fine motor coordination, as measured on the grooved pegboard task, was low average for the dominant side $(T=40)$, although average for the nondominant side $(T=49)$. No abnormal laterality signs were noted. No akinetic or perseverative tendencies were evident.

\section{Language Functions}

On incidental behavior, mild phoneme imprecision was evident and use of verb tense and plural nouns were frequently incongruent. Nevertheless, spontaneous speech was fluent, grammatically adequate, and conveyed information satisfactorily. Comprehension of questions and serial commands was within normal limits, although he did err in comprehension of irregular semantic questioning. Repetition of high and low frequency phrases was normal. Confrontational naming (Boston Naming Test) was significantly impaired compared to other males his age. However, no paraphasic errors or circumlocutions were noted and his performance did not significantly differ from the score predicted from his level of reading achievement (Hawkins et al., 1993).

\section{VisuoSpatial Functions}

Copy of a simple Greek cross was within normal limits. In contrast, reproduction of a complex line drawing was significantly impaired (more than 4 standard deviations below the mean). Visual discrimination was mildly impaired $(T=37)$, although complex visual integration was average (Hooper: $T=46$ ).

Mr. Dexter's drawing of a clock was borderline impaired because of indistinguishable hour and minute hands and mild rotation in numeral placement. On a time-setting task, the hands of the clock were again indistinguishable, but they pointed to the correct numerals. One error (in a trial of four items) in time reading was made, mistaking 9:00 for 3:00, further suggesting difficulty in distinguishing the hour and minute hands on analog clocks.

Identification of visual details was below average $(T=40)$ and mild impairment was evidenced on three dimensional block constructions $(T=37)$. Mild to moderate impairment was demonstrated on sequencing of everyday events from visual cues $(T=33)$ and nonverbal abstract reasoning $(T=30)$. Mr. Dexter's WAIS-III Perceptual Organization Index score fell in the borderline impaired range (Index score: 74).

\section{Memory Functions}

Mr. Dexter demonstrated significantly impaired incidental visual memory for a complex line drawing (Rey-O) during both immediate free recall and recall following short delay 
Table 1

Wechsler Memory Scale-III Scores on the Primary Indexes

\begin{tabular}{lccccc}
\hline $\begin{array}{l}\text { Wechsler Memory Scale-III } \\
\text { Primary Indexes }\end{array}$ & $\begin{array}{c}\text { Standard } \\
\text { Score }\end{array}$ & $\begin{array}{c}\text { 95\% Confidence } \\
\text { Interval }\end{array}$ & $T$ Score & $\begin{array}{c}\text { Percentile } \\
\text { Rank }\end{array}$ & $\begin{array}{c}\text { Qualitative } \\
\text { Description }\end{array}$ \\
\hline Auditory immediate & 50 & $46-60$ & 17 & $<1 \mathrm{st}$ & Impaired \\
Visual immediate & 65 & $61-81$ & 26 & $1 \mathrm{st}$ & Impaired \\
Immediate memory & 47 & $44-59$ & 14 & $<1 \mathrm{st}$ & Impaired \\
Auditory delayed & 55 & $52-70$ & 20 & $<1 \mathrm{st}$ & Impaired \\
Visual delayed & 56 & $53-73$ & 21 & $<1 \mathrm{st}$ & Impaired \\
Auditory recognition & 75 & $70-93$ & 33 & 5 th & Borderline \\
$\quad$ delayed & & & & & impaired \\
General memory & 52 & $48-64$ & 18 & $<1 \mathrm{st}$ & Impaired \\
Working memory & 57 & $54-72$ & 21 & $<1 \mathrm{st}$ & Impaired \\
\hline
\end{tabular}

(more than 4 standard deviations below the mean on each occasion). Table 1 summarizes Mr. Dexter's performances on the WMS-III. On the WMS-III, Mr. Dexter demonstrated impaired single-trial learning and a poor learning curve $(T \mathrm{~s}=26)$ for information presented verbally. Immediate spontaneous recall was impaired for both verbal $(T=17)$ and visual $(T=26)$ information. Free recall of both verbal and nonverbal material after 30-minute delay was also impaired ( $T \mathrm{~s}=20$ and 21 , respectively). Delayed recognition was significantly better, although still poor $(T=33)$, suggesting that Mr. Dexter experiences difficulty retrieving information rather than rapid forgetting per se. With the single exception of his delayed auditory recognition, Mr. Dexter's obtained memory scores are significantly lower (at the .01 level) than predicted based on his overall intellectual abilities. However, no perseverative contaminations or confabulations were noted.

\section{Intellectual Functioning}

Mr. Dexter obtained a full scale IQ on the WAIS-III that falls within the borderline impaired range (SS: 73; fourth percentile). No statistically significant difference was evident between his obtained Verbal IQ (SS: 77; sixth percentile) and Performance IQ (SS: 73; fourth percentile). Table 2 presents Mr. Dexter's subtest scores on the WAIS-III, whereas Table 3 presents his index and IQ scores.

Examination of the index scores reveals a statistically significant difference between Mr. Dexter's obtained Working Memory Index $(T=28)$ and Verbal Comprehension Index $(T=38)$. This significant difference in broad abilities is partially reflective of Mr. Dexter's evidencing a relative strength in his knowledge of word definitions $(T=43)$, although this area is still slightly below average. Furthermore, the index score discrepancy was adversely affected by Mr. Dexter's demonstration of a specific, relative weakness in a more complex task that requires manipulation of letters and numbers in working memory $(T=26)$.

\section{Self-Regulation and Executive Functions}

No asocial, akinetic, perseverative, or impulsive behaviors were observed in incidental behavior. Mr. Dexter performed in the mildly to moderately impaired range for both phonemic 
Table 2

Obtained Wechsler Adult Intelligence Scale-III Subtest Score

\begin{tabular}{lccc}
\hline WAIS-III Subtest & Age Scaled Score & $T$ Score & Percentile Rank \\
\hline Digit symbol & 6 & 37 & 9 th \\
Symbol search & 3 & 26 & 1 st \\
Arithmetic & 5 & 33 & 5 th \\
Digit span & 6 & 37 & 9 th \\
Letter-number sequencing & 3 & 26 & 1 st \\
Vocabulary & 8 & 43 & 25 th \\
Similarities & 7 & 40 & 16 th \\
Comprehension & 6 & 37 & 9 th \\
Picture completion & 7 & 40 & 16 th \\
Block design & 6 & 37 & 9 th \\
Matrix reasoning & 4 & 30 & 2 nd \\
Picture arrangement & 5 & 33 & 5 th \\
\hline
\end{tabular}

Table 3

Obtained Wechsler Adult Intelligence Scale-III Index and IQ Scores

\begin{tabular}{lcccrl}
\hline Index & $\begin{array}{c}\text { Index } \\
\text { Score }\end{array}$ & $\begin{array}{c}\text { 95\% Confidence } \\
\text { Interval }\end{array}$ & $T$ Score & $\begin{array}{c}\text { Percentile } \\
\text { Rank }\end{array}$ & $\begin{array}{c}\text { Qualitative } \\
\text { Description }\end{array}$ \\
\hline Processing speed index & 71 & $66-83$ & 30 & 3 rd & Borderline impaired \\
Working memory index & 67 & $62-76$ & 28 & 1 st & Impaired \\
Verbal comprehension index & 82 & $77-88$ & 38 & 12th & Low average \\
Perceptual organization index & 74 & $69-83$ & 33 & 4 th & Borderline impaired \\
Verbal IQ & 77 & $73-83$ & 34 & 6th & Borderline impaired \\
Performance IQ & 73 & $68-81$ & 32 & 4th & Borderline impaired \\
Full scale IQ & 73 & $69-78$ & 32 & 4 th & Borderline impaired
\end{tabular}

fluency $(T=33)$ and semantic fluency $(T=34)$, with no significant incidence of inappropriate or perseverative errors. Rhythm tapping was within normal limits, but complex motor programming revealed mild incoordination and some loss of mental set. He performed within normal limits on a go/no-go task during trials with habitual meaning; however, inhibition on motor go/no-go with reversed meaning was mildly impaired. Performance on a planning task (WISC-III Mazes: $T=30$ ) was mildly to moderately impaired.

On a timed task that required the simultaneous visual tracking of two lines of thought, Mr. Dexter performed in the impaired range, due to slowing. On an auditory task that similarly required the simultaneous tracking of two lines of thought, while inhibiting competing responses, Mr. Dexter performed within the mildly to moderately impaired range (Stroop Color-Word: $T=34$ ). Each of these performances may have been somewhat adversely affected by Mr. Dexter's relatively weak speed of information processing, due to the timed nature of the tasks. 


\section{Adaptive Behavior Skills}

Mr. Dexter was asked to rate his abilities in nine adaptive skill areas across three domains (e.g., conceptual, practical, and social). His self-report of his general adaptive functioning fell in the mildly to moderately impaired range $(T=34)$, without significant scatter among the conceptual $(T=30)$, social $(T=33)$, and practical $(T=38)$ domains. With the exception of the home living skills domain, the scores derived from Mr. Dexter's self-report are within the expected range based on his general intellectual abilities. His score for home living skills is higher than expected, although it is possible that Mr. Dexter portrayed his abilities in a slightly flattering light.

\section{Functional Academic Skills}

On a task of word recognition, Mr. Dexter performed in the mildly to moderately impaired range ( $T=30 ; 3 \mathrm{rd}$ percentile), which is approximately equivalent to achievement at the fourth-grade level. Similarly, he demonstrated mildly impaired achievement on a test of spelling $(T=39 ; 14$ th percentile), which is approximately equivalent to achievement at the sixth-grade level. On a task of written arithmetic skills, Mr. Dexter scored in the moderately to severely impaired range ( $T=22 ;<1$ st percentile), which is approximately equivalent to achievement at the third-grade level. Stated another way, $97 \%$ of adults perform better on word recognition, $86 \%$ of adults perform better on spelling, and more than $99 \%$ of adults perform better on written arithmetic in comparison to Mr. Dexter.

\section{Emotional Status and Psychopathology}

The Personality Assessment Inventory was administered to Mr. Dexter with the examiner reading the questions to him because his word recognition skills are at the lower limit necessary for completion of the test. This may have affected his response style, although the validity indices were within normal limits. Clinical elevations suggested that Mr. Dexter was experiencing considerable distress including a broad range of depressive symptoms, thoughts of death or suicide, and psychotic symptoms. Poor anger control with a history of antisocial behavior was acknowledged and adverse impact from traumatic events was indicated by the pattern of responding. Finally, considerable difficulties related to drug dependence were reported, at a level that exceeds that usually observed in those seeking treatment for such problems.

\section{Case Conceptualization}

Mr. Dexter was first evaluated shortly after his formal education was initiated in middle to late childhood. Records from that evaluation documented significant intellectual impairment and an educational classification of mental retardation was recommended as a result. With specialized education experiences during the next 3 years, Mr. Dexter was able to improve to the low average range of general intellectual abilities. He was no longer considered mentally retarded by the school system, but focal persisting deficiencies were recorded and he was identified as having a mathematics-related learning disorder. 
During the current evaluation, formal testing of Mr. Dexter's intellectual abilities revealed impaired to borderline impaired general intellectual functioning with no clinically or statistically significant difference between his broad verbal and nonverbal abilities. Neurocognitive testing revealed corresponding significant impairments in a range of abilities including attention and concentration, processing speed, language, and memory. In contrast, Mr. Dexter's motor abilities were within normal limits and his basic visual sensory and perceptual functioning was intact. Spatial abilities, more generally, were variable and ranged from impaired to normal.

Mr. Dexter's self-reported adaptive functioning was consistent with his broad cognitive functioning, with significant impairments in three skills areas (i.e., communication, health and safety, and leisure). In addition, formal testing of functional academic skills revealed impaired achievement in word recognition, spelling, and arithmetic. Given his current functioning, he is unlikely to be successful in unsupervised living or employment settings because of his deficient skills. Significant psychopathology, including psychotic symptoms, was also evident and such features are likely to further undermine Mr. Dexter's functioning.

Although Mr. Dexter's scores during this evaluation are largely supportive of mental retardation, his low average performance at the time of his last evaluation in childhood is inconsistent with this conceptualization. If the results of the last testing session in childhood are accurate, the results of the current evaluation suggest a clinically and statistically significant decline in Mr. Dexter's intellectual abilities that is consistent with a DSM-IV-TR (American Psychiatric Association, 2000) diagnosis of 294.9 Cognitive Disorder Not Otherwise Specified.

\section{Complicating Factors}

Interpretation of the data is complicated in several regards. First, Mr. Dexter's full-scale IQ (73) poses difficulty in determining whether a mental retardation classification is appropriate. The $95 \%$ confidence range (69 to 78) indicates that Mr. Dexter's true abilities fall somewhere in the impaired to borderline impaired range. However, neither the DSM-IV-TR (American Psychiatric Association, 2000) nor the AAMR (1983, 1992, 2002) definitions of mental retardation provide specific principles on the interpretation of such ambiguous scores instead suggesting that the examiner consider adaptive functioning and behavioral observations to determine whether the overall pattern is consistent with mental retardation. However, with the Atkins v. Virginia (2002) ruling, this lack of specificity poses difficulty in legal proceedings with experts drawing different conclusions about whether such an individual is properly classified as mentally retarded, even as the person's life is at stake.

Review of the testing records obtained during Mr. Dexter's childhood and adolescence revealed that he underwent evaluation three times during approximately 3 years. If his scores on the first evaluation are used as a baseline for comparison with the present scores, then classification of Mr. Dexter as mentally retarded seems appropriate. However, if the scores from his second childhood testing are used as the baseline, it becomes quite ambiguous with both his childhood full-scale IQ and his present full-scale IQ falling near the lower limit of the borderline impaired range. Finally, Mr. Dexter's best scores, obtained at the last testing session during childhood, fall in the low average range and do not lend support to a 
conceptualization of mental retardation. The improvement in scores over time might cause one to speculate on the possible effects of practice. However, review of the data from those evaluations indicates that the evaluators gave some consideration to this risk and used varying measures to test Mr. Dexter's cognitive abilities and academic achievement. Thus, it appears that Mr. Dexter's low average abilities at the final testing session in childhood was reasonable, with the previously demonstrated impairment reflecting impoverishment from not attending school for several years during early and middle childhood.

If Mr. Dexter did have low average general cognitive abilities prior to the age of 18, the current findings are lower than expected and the question of malingering is raised. However, if Mr. Dexter was attempting to simulate mental retardation, he failed to perform unambiguously impaired during intelligence testing, which is in contrast to the findings reported by Johnstone and Cooke (2003) who noted that individuals simulating impairment tended to perform so poorly that floor effects emerged in the obtained intelligence profiles. Although not a test of intelligence malingering, Mr. Dexter was able to perform within normal limits on a TOMM that counters a broad malingering hypothesis.

The question of malingering is not fully resolved, however. Mr. Dexter's immediate memory for both verbal and visual information, as measured by the WMS-III, is significantly below the values predicted based on his WAIS-III scores. Given Mr. Dexter's extremely limited formal education, demographically corrected norms were therefore considered desirable. The usage of such norms would offer clarification as to whether Mr. Dexter's obtained WMS-III scores really were below expectations or an artifact of his limited formal education. However, these adjusted norms are only available for Caucasian, Hispanic, and African American groups, with no corrections available for Native American individuals.

Personal communication with a technician for the Wechsler scales publisher (R. Belton, personal communication, January 17, 2006) indicated that during the WAIS-III standardization phase, too few Native American participants were recruited to allow for demographic corrections to be formulated. In fact, examination of standardization data revealed that only 5 of the 247 participants $(.02 \%)$ with full-scale IQs of equal to or less than 80 were classified as something other than Black, White, or Hispanic with no specific classification at all for Native American, although the prevalence of Native Americans within the United States is approximately $1.5 \%$ (Ogunwole, 2006). Similarly, examination of the standardization data determined that only 5 of 115 participants (.04\%) with SSs of 80 or less on all 9 adaptive functioning subtests of the Adaptive Behavior Assessment System (Harrison \& Oakland, 2000) were classified as other ethnicity. Such demographic corrections would be useful because Census 2000 findings report that the Native American educational levels were lower than the total population levels (Ogunwole, 2006), although variability among tribal groupings was observed.

Assuming that the last testing session in childhood and the current results of testing are reliable, the etiology of Mr. Dexter's apparent cognitive decline cannot be determined based solely on the results of testing and may be multifactorial. For example, Mr. Dexter may have experienced some hypoxia (i.e., oxygen deprivation that can result in brain injury; Gale, Hopkins, \& Weaver, 1999; Kesler, Hopkins, \& Weaver, 2001; Porter, Hopkins, \& Weaver, 2002) as a result of his carbon monoxide inhalation and respiratory distress following the house fire. In addition, he described a lengthy history of largely untreated psychotic symptoms, which has been linked to cognitive decline in the research literature (Johannessen, 
Larsen, \& McGlashan, 1999; McGlashan, 1999). He also has a lengthy history of substance abuse and dependence, which included huffing gas. Such behavior is linked to neurotoxicity and decline (Scelsa, 2000; see Hannay, Howieson, Loring, Fischer, \& Lezak, 2004, for a broad overview of cognition and substance abuse). Finally, at the time of the present evaluation, Mr. Dexter had been residing in solitary conditions for an extended period of time that also may have adversely affected his current functioning (Houston, 1967).

\section{Recommendations for Follow-Up}

Mr. Dexter's ability to learn new information is significantly impaired and poses a barrier to success in future learning opportunities. However, his recognition memory is substantially better than his free recall, suggesting that multiple-choice presentations may be a useful format for assessing his acquisition of new information if future learning or rehabilitation interventions are undertaken by Mr. Dexter.

Significant psychopathology, including psychotic symptoms, was evident, and these features are likely to undermine Mr. Dexter's functioning. As a result, continued psychiatric care was strongly urged with ongoing monitoring of the effectiveness of interventions. More specifically, careful follow-up by a mental health professional regarding the details of Mr. Dexter's suicidal thoughts and the potential for suicidal behavior was recommended with appropriate interventions, including suicide precautions, encouraged without delay if the potential for suicide was confirmed. Although the solitary conditions might have been adversely affecting Mr. Dexter's cognitive functioning, command auditory hallucinations encouraging violence contraindicated any recommendation for a less restrictive environment.

\section{Implications of the Case}

The primary implication this case is intended to illustrate is the need for standardization efforts of tests to include adequate participant representation and better classification of race. Although this may necessitate challenges posed by focused recruitment efforts and potentially lengthen the product development to market timeline, such care is warranted. With the Atkins v. Virginia (2002) ruling, psychologists are likely to be involved in high stakes assessment of mental retardation. The absence of appropriate normative information is problematic and increases the likelihood of experts' failing to agree on the interpretation of findings. Clearly, such conflicts are undesirable in such cases when making the proper interpretation has very real life or death consequences. More generally, as a modifiable variable, failing to appropriately and comprehensively standardize popular measures used in evaluative settings does not further the public's perception of the field of psychology.

An additional clinical implication is the need for the development of measures to assess for malingering of mental retardation. Such a measure would, ideally, measure not only intellectual ability but also adaptive functioning domains. Consideration of the likelihood that some individuals will be repeatedly assessed by different examiners within an undesirably short time span should also be made by developing psychometrically sound parallel forms of both malingering measures and measures of cognition and adaptive functioning. 
Additional research into the simulation of mental retardation on those measures presently available is also necessary. In the wake of the Atkins v. Virginia (2002) ruling, it is possible that some individuals will become educated about commonly used tests and use this information to better simulate mental retardation. Although the Johnstone and Cooke (2003) study provides some information as to how flagrantly simulated mental retardation is reflected on intellectual measures, additional research examining more sophisticated simulations and how they differ from true profiles is necessary. To that end, further research examining the cognitive and adaptive profiles of individuals known to have mental retardation is needed.

Self-report of adaptive functioning is ideally corroborated with other sources, although in this case, the lack of reliable informants was an obstacle. Unfortunately, no studies are located in the literature examining adaptive functioning simulation as it might pertain to identifying malingering mental retardation. This is a very important area for consideration in both clinical practice and in future research. Clinical lore has indicated that individuals who are truly mentally retarded might overestimate personal functioning as a result of poor insight or glorify past achievements to ward off stereotypic threat (see Steele, 1997, for an example of the relationship between stereotypic threat and intellectual identity development). It seems reasonable to hypothesize that individuals feigning mental retardation would be motivated to simulate deficits but this should be examined in research. In the current evaluation, Mr. Dexter did report deficiencies in the three broad areas of adaptive functioning, but examination of the individual subtests revealed discrete areas of strength, with home living skills reportedly average; this appears to be consistent with research indicating that persons with mental retardation often display both strengths and weaknesses (AAMR, 2002). Studies that examine simulation of deficits on both self-report and performancebased measures of adaptive functioning are urgently needed.

\section{Recommendations to Clinicians and Students}

Because individuals being assessed may undergo repeated testing, it is important for examiners to determine whether the individual being assessed has familiarity with the measures being used. When testing within a forensic setting this poses some complications because secure settings typically require a detailed listing of materials in advance of the evaluation so that they can be inventoried on arrival to the secure setting. Clinicians entering into such evaluations should anticipate extensive record review and the need to track down historical records before conducting the evaluation. Individuals should also be acquainted with the applicable state legislation prior to initiating the evaluation as well as professional literature outlining current issues with such evaluations and the relevant competencies necessary (e.g., Bonnie, 2004).

\section{References}

American Association on Mental Retardation. (1983). Mental retardation (8th ed.). Washington, DC: Author. American Association on Mental Retardation. (1992). Mental retardation (9th ed.). Washington, DC: Author. American Association on Mental Retardation. (2002). Mental retardation (10th ed.). Washington, DC: Author. 
American Psychiatric Association. (2000). Diagnostic and statistical manual of mental disorders-text revision (4th ed.). Washington, DC: Author.

Atkins v. Virginia, 526 U.S. 304 (2002).

Bonnie, R. J. (2004). The American Psychiatric Association's resource document on mental retardation and capital sentencing: Implementing Atkins v. Virginia. Journal of the American Academy of Psychiatry and the Law, 32, 304-308.

Gale, S. D., Hopkins, R. O., \& Weaver, L. K. (1999). MRI, quantitative MRI, SPECT and neuropsychological findings following carbon monoxide poisoning. Brain Injury, 13, 229-243.

Hannay, H. J., Howieson, D. B., Loring, D. W., Fischer, J. S., \& Lezak, M. D. (2004). Neuropathology for neuropsychologists. In M. D. Lezak, D. B. Howieson, \& D. W. Loring (Eds.), Neuropsychological assessment (4th ed., pp. 157-285). New York: Oxford University Press.

Harrison, P. L., \& Oakland, T. (2000). Adaptive behavior assessment system. San Antonio, TX: Psychological Corporation.

Hawkins, K. A., Sledge, W. H., Orleans, J. F., Quinland, D. M., Rakfeldt, J., \& Hoffman, R. E. (1993). Normative implications of the relationship between reading vocabulary and Boston Naming Test performance. Archives of Clinical Neuropsychology, 8, 525-537.

Houston, S. H. (1967). Inquiry into the structure of mentation processes. Psychological Reports, 21, 649-659.

Johannessen, J. O., Larsen, T. K., \& McGlashan, T. (1999). Duration of untreated psychosis: An important target for intervention in schizophrenia? Nordic Journal of Psychiatry, 53, 275-283.

Johnstone, L., \& Cooke, D. J. (2003). Feigned intellectual deficits on the Wechsler Adult Intelligence Scale-Revised. British Journal of Clinical Psychology, 42, 303-318.

Kesler, S. R., Hopkins, R. O., \& Weaver, L. K. (2001). Verbal memory deficits associated with fornix atrophy in carbon monoxide poisoning. Journal of the International Neuropsychological Society, 7, 640-646.

McGlashan, T. (1999). Duration of untreated psychosis in first-episode schizophrenia: Marker or determinant of course? Biological Psychiatry, 46, 899-907.

Ogunwole, S. U. (2006). We the people: American Indians and Alaska Natives in the United States. Washington, DC: U.S. Census Bureau.

Porter, S. S., Hopkins, R. O., \& Weaver, L. K. (2002). Corpus callosum atrophy and neuropsychological outcome following carbon monoxide poisoning. Archives of Clinical Neuropsychology, 17, 195-204.

Rey, A. (1964). L'examen clinique en psychologie [Clinical assessment in psychology]. Paris: Presses Universatairies de France.

Scelsa, S. N. (2000). Nitrous oxide. In P. S. Spencer \& H. H. Schaumburg (Eds.), Experimental and clinical neurotoxicology (pp. 882-888). New York: Oxford University Press.

Steele, C. M. (1997). A threat in the air: How stereotypes shape the intellectual identities and performance of women and African-Americans. American Psychologist, 52, 613-629.

Tombaugh, T. N. (1996). Test of memory malingering. Los Angeles, CA: Western Psychological Services.

Wechsler, D. (1997a). The Wechsler Adult Intelligence Scale (3rd ed.). San Antonio, TX: Psychological Corporation.

Wechsler, D. (1997b). The Wechsler Memory Scale (3rd ed.). San Antonio, TX: The Psychological Corporation.

Jennifer L. Callahan, $\mathrm{PhD}$, is an assistant professor of psychology at Oklahoma State University, Stillwater, Oklahoma. She also holds a visiting appointment at Yale University School of Medicine in the Department of Psychiatry, New Haven, Connecticut. She is the director of the Psychological Services Center, a training clinic for doctoral students in clinical psychology at Oklahoma State University. Her research interests span the assessment (clinical and neuropsychological) and psychotherapy domains. 